\title{
Critical assessment of charge transfer estimates in non-covalent graphene doping
}

\author{
Iann C. Gerber* and R. Poteau \\ LPCNO, Université Fédérale de Toulouse Midi-Pyrénées, \\ INSA-CNRS-UPS,135 Av. de Rangueil, 31077 Toulouse, France
}

\begin{abstract}
Non covalent doping by pure charge transfer complexes is one possible solution to tune at low cost electronic properties of carbon based nano-structures, more specifically to enhance their conductivity. Here we present a thorough density functional theory based study of charge transfer estimates, by comparing available integration/partitioning scheme of the electronic density in periodic boundary conditions, as well as the influence of the exchange-correlation term, the cornerstone of DFT by testing various exchange-correlation functionals. Our test case is made of a freestanding graphene monolayer in interaction with two prototypical donor/acceptor molecules: TTF and TCNE. These results illustrate the role played by the exact-exchange in the description of charge transfer processes, as well as the difference between the density-based and wavefunction-based partitioning schemes used in this study. When using hybrid functionals, charge transfer are usually smaller than when using standard generalized gradient approximations, especially for the donor molecule. In terms of electronic density partitioning schemes, both strategy provide quite similar charge transfers, however each intra-molecular decomposition presents very distinct features, making the discussion of atomic charge re-organisation on the electron/donor molecule highly dependent of the selected partitioning scheme.
\end{abstract}

*Electronic address: igerber@insa-toulouse.fr 


\section{INTRODUCTION}

Graphene, a single exfoliated layer of graphite, was first obtained by the group of Novoselov [1]. Due to its special honeycomb structure, implying only $s p^{2}$ carbon atoms, the valence band (VB) and the conduction band (CB) intersect at the Dirac point. Interestingly the energy-momentum dispersion is linear around the Dirac point [2]. As a result ballistic charge transport has been observed [3], and ideally this property could be useful in wide range of applications. Therefore, graphene-based devices have been considered as a promising alternative to conventional silicon-based ones. By shifting the Fermi level relative to the Dirac point, the carrier type and concentration in graphene can be controlled through electrical doping either with an external electric field $[1,4,5]$ or chemical doping with different atoms and/or molecules, see Ref. [6-9] and references therein. Among all the possibilities offered by a chemical functionalization of a graphene, the physisorption of some electron donor/acceptor molecules is particularly simple, non-destructive and effective $[10,11]$. The mechanism of charge transfer (CT) between electron donor/acceptor (EDA) and a graphene monolayer has been elucidated by one of the author [12], completed by a later study [13]. CT occurs as soon as a difference in electronic chemical potentials is present at the interface. This difference is thus simply determined by the relative positions of the graphene Fermi level and the highest occupied (HOMO) and lowest unoccupied (LUMO) molecular orbitals of the adsorbate. If the LUMO of the adsorbate lies lower in energy than the Fermi level of graphene, electrons will flow from graphene to the adsorbate making graphene doped by holes, i.e p-type. On contrary adsorbates with the HOMO lying above the graphene Fermi level will act as donors, thus n-type doping graphene.

Theoretically speaking it means that to be predictive and quantitative, a DFT study of the interaction between any electron donor/acceptor molecule and graphene, needs to include (i) a correct description of the electronic structure of the molecule, more specifically to put the HOMO/LUMO energy at the right position and (ii) since the adsorbate is only physisorbed, a correct description of the van der Waals forces is mandatory, to put the molecule at the right distance above graphene. Indeed in the work of $\mathrm{Hu}$ et al it has been shown that $\mathrm{CT}$ is crucially dependent of the distance between the EDA molecule and graphene [12]. The main concern of all the previous studies in this field were devoted to theoretically determine the existing CT, mainly thanks to the "Quantum Theory of Atoms in Molecules" (QTAIM), 
that is able to partition the electronic density based on topological arguments [14]. Mind that vdW interactions were not systematically included in previous works dealing with the interaction between organic EDA molecule with graphene [15-24]. Thus it is challenging to correctly describe CT complexes usually since (i) the local density approximation (LDA) or generalized gradient approximations (GGA) of the exchange-correlation functionals tend to over or under-bind respectively, (ii) the position of the HOMO or LUMO adsorbate with respect to the Fermi level of graphene may be erroneous. We propose here, to investigate the use of hybrid functionals, which provide electronic structures, especially HOMO-LUMO gap, in better agreement with experimental data, to estimates CT for two prototypical EDA molecules, the electron-acceptor TCNE (tetracyano-ethylene) and the electron donor TTF (tetrafulvalene) in interaction with graphene. We also compare different methods for the projections/partitioning of the electronic densities, to quantify the CT.

\section{COMPUTATIONAL DETAILS}

The atomic structures have been obtained from DFT calculations using the VASP package. $[25,26]$ It uses the projector-augmented wave scheme $[27,28]$ to treat core electrons. The $2 s$ and $2 p$ states of carbon and nitrogen, $1 s$ state of hydrogen and $3 s$ and $3 p$ states of sulfur have been treated explicitly in the valence. The graphene model used is made of $(7 \times 7)$ primitive cell, with a relaxed C-C bond-length of $1.42 \AA$. As usual in this type of calculations, we have applied a supercell approach, with a vacuum region of more than $16 \AA$ to avoid interaction between periodic images in the perpendicular direction. On top of this substrate one single molecule (TCNE or TTF) has been absorbed, in various sites of high symmetry using the notations of a previous work [12]. All atoms were allowed to relax with a force convergence criterion below $0.005 \mathrm{eV} / \AA$, and to ensure a reliable distance between the adsorbate and graphene, we have accounted for van der Waals interactions through the use of opt-B86b-DF scheme [29, 30] for every geometry optimization runs. The plane-wave basis set cutoff energy was set to $400 \mathrm{eV}$ with a Gaussian smearing method of $0.1 \mathrm{eV}$ width, in order to assure well converged total energy and force values, in conjunction with a $(3 \times 3 \times 1) \Gamma$-centered k-point grid. Choice of the exchange-correlation functional has been tested to estimate CT, by using spin polarized version of the Generalized Gradient Approximation (GGA) PBE [31], two hybrid functionals PBE0 [32, 33] and HSE [34-36], 
as well as RSHXLDA scheme [37, 38] known to produce correct long-range exchange behavior. Bader charge analysis, based on Atoms in Molecule theory [14], were carried out using Henkelman's Group program [39-41], after a careful checking of the integration grid dimension controlled by the Fast Fourier Grid parameters. Those charges have been compared with the ones from a Mulliken-like population analysis (MPA) within VASP. Indeed it is well known that absolute partial charge values can vary signicantly between different partitioning schemes. [42, 43] MPA from VASP wavefunction was performed by integrating up to the Fermi energy the Density of States projected onto an atomic Slater basis set (pDOS). These charges are hereafter mentioned as pMPA charges. The projection of the PAW wavefunction was achieved with the Lobster software, using the pbeVASPfit basis set [44-46]. The charge spilling, a criterion that assesses the quality of the projection, was systematically lower than 1.0\%. Various electronic density decomposition schemes (MPA, AIM-Bader, Natural Population Analyzis [47], CM5 [48]) were also applied to TCNE and TTF molecules alone, using the $6-311++\mathrm{G}(d, p)$ Gaussian basis sets within the G09 software [49]. These wavefunction analysis were carried out with the Multiwfn software [50].

\section{RESULTS}

\section{A. Free compounds}

Several atomic charges calculation methods are first going to be evaluated on the gasphase TCNE and TTF molecules. Two procedures based on molecular orbitals were examined - i.e. in the framework of Gaussian atomic basis sets - the Mulliken population analysis (MPA) and the Natural population analysis (NPA). They have been compared to two methods based on the density distribution: the Bader's Atom In Molecule (AIM), also known as Bader charges, and the Charge Model 5 (CM5), which is an extension of Hirshfeld population analysis. AIM charges were also calculated from the density functional electronic charge distributions calculated in a pseudopotential - plane waves basis set, under periodic boundary conditions, i.e. with a supercell approach. Molecular orbitals based methods cannot be directly used with such methods, both because a density matrix in a localized atom-centered basis set is required, and because the electronic wave function for periodic systems is given in reciprocal space, thus yielding electronic states delocalized over all space. 


\begin{tabular}{c|ccc|ccc}
\hline & \multicolumn{3}{|c|}{ PBE } & \multicolumn{3}{c}{ PBE0 } \\
& $\mathrm{C}(=\mathrm{C})$ & $\mathrm{C}(\equiv \mathrm{N})$ & $\mathrm{N}$ & $\mathrm{C}(=\mathrm{C})$ & $\mathrm{C}(\equiv \mathrm{N})$ & $\mathrm{N}$ \\
\hline \hline MPA $^{\mathrm{a}}$ & 2.29 & -1.03 & -0.11 & 2.46 & -1.10 & -0.13 \\
pMPA $^{\mathrm{b}}$ & -0.18 & 0.37 & -0.27 & -0.17 & 0.37 & -0.27 \\
$\mathrm{NPA}$ & -0.15 & 0.25 & -0.17 & -0.14 & 0.25 & -0.18 \\
AIM $^{\mathrm{a}}$ & 0.16 & 0.83 & -0.91 & 0.18 & 0.91 & -1.00 \\
AIM $^{\mathrm{b}}$ & 0.25 & 0.87 & -0.99 & 0.31 & 0.89 & -1.05 \\
$\mathrm{CM}$ & 0.08 & 0.20 & -0.24 & 0.09 & 0.21 & -0.25 \\
\hline
\end{tabular}

TABLE I: Partial atomic charges in TCNE (in units of $e$ ). ${ }^{\mathrm{a}}$ : Gaussian basis set (G09); ${ }^{\mathrm{b}}$ : PW basis set (VASP).

A way to circumvent these fundamental challenges is to employ a plane waves to atomic orbitals projection scheme. This is the strategy used in the present study, taking advantage on a projected density of states, from which an MPA can be easily achieved. It must be underlined that since the resulting charges, referred to as pMPA charges, are obtained within an orthonormal minimal Slater basis set, they are not expected to be similar to the Gaussian atomic basis sets MPA charges. On top of that, the PBE GGA functional will be compared to the PBE0 hybrid functional. The comparison of the AIM or pMPA charges in these free compounds with the complexes will then show where the electron density depletion or increase does occur upon an intermolecular CT phenomenon.

TCNE molecule. The first general comment is that PBE0 and PBE atomic charges are very close, whatever the method (table I). The explicit exchange contribution in the functional does not significantly affect this property. Although basis sets and pseudopotentials differ between VASP and G09, AIM charges are found to be almost the same, thus showing a weak basis set dependency of this density partitioning scheme. Not surprisingly, MPA and pMPA charges strongly differ from each other. It is a good point for pMPA, since MPA atomic charges are questionable, with a 2.29e (PBE) or 2.46e (PBE0) very strong charge on the ethylenic carbon atoms and a strongly negative charge on the cyano groups carbon atoms (PBE: -1.03e, PBE0: -1.10e). Interestingly, it can be seen from table I that pMPA and NPA charges are very similar, whereas CM5 charges are to some extent found to be closer to pMPA and NPA than to AIM. All methods agree that nitrogen atoms are 


\begin{tabular}{c|cccc|cccc}
\hline & \multicolumn{5}{|c|}{ PBE } & \multicolumn{5}{c}{ PBE0 } \\
& C(central) & C(lateral) & $\mathrm{H}$ & $\mathrm{S}$ & $\mathrm{C}($ central $)$ & C(lateral) & $\mathrm{H}$ & $\mathrm{S}$ \\
\hline \hline MPA $^{\mathrm{a}}$ & 0.63 & -0.12 & 0.26 & -0.45 & 0.66 & -0.12 & 0.27 & -0.48 \\
pMPA $^{\mathrm{b}}$ & -0.51 & -0.57 & 0.37 & 0.46 & -0.49 & -0.56 & 0.37 & 0.45 \\
NPA & -0.45 & -0.40 & 0.24 & 0.38 & -0.44 & -0.39 & 0.24 & 0.37 \\
AIM $^{\mathrm{a}}$ & -0.22 & -0.15 & 0.09 & 0.17 & -0.24 & -0.16 & 0.09 & 0.18 \\
AIM $^{\mathrm{b}}$ & -0.24 & -0.20 & 0.14 & 0.18 & -0.24 & -0.20 & 0.15 & 0.17 \\
$\mathrm{CM}^{5}$ & -0.05 & -0.11 & 0.12 & 0.02 & -0.04 & -0.11 & 0.12 & 0.01 \\
\hline
\end{tabular}

TABLE II: Partial atomic charges in TTF (in units of $e$ ). ${ }^{\mathrm{a}}$ : Gaussian basis set (G09); ${ }^{\mathrm{b}}$ : PW basis set (VASP).

negatively charged, with a $c a$. $-1 e$ charge with AIM and a $-1 / 4 e$ or $-1 / 5 e$ charge with the other methods. Whereas the global charge of cyano groups is found to be slightly negative with AIM and CM5 (between -0.04e and -0.1e), it is slightly positive with pMPA and NPA $(\sim 0.1 e)$. As a consequence ethylenic carbon atoms exhibit a negative charge according to pMPA and NPA and a positive charge with AIM and CM5, which is apparently more in line with the electron-withdrawing character of cyano groups. However, what is more important is the relative value of atomic charges upon the replacement of a functional group, a trend well accounted for by pMPA and NPA when comparing to ethylene $(-0.50 e$ and $-0.38 e$ respectively).

TTF molecule. As observed previously with TCNE, PBE0 and PBE charges are very similar (table II). MPA again provides very different charges from the other methods, and pMPA and NPA charges are still very close. Whereas in TCNE the AIM charges were close to unity on the $\mathrm{C}$ and $\mathrm{N}$ atoms of the cyano groups, no large intermolecular charge transfer is found here with this method. On the contrary, it is now the pMPA and NPA methods that provide the highest charge values. It is worth mentioning that CM5 charges are close to zero, in contrast with the other procedures. pMPA, NPA and AIM actually qualitatively agree, with positive sulfur atoms, negative central carbon atoms and a slightly positive $\mathrm{C}_{2} \mathrm{H}_{2}$ moiety. 


\section{B. Charge Transfer Complexes}
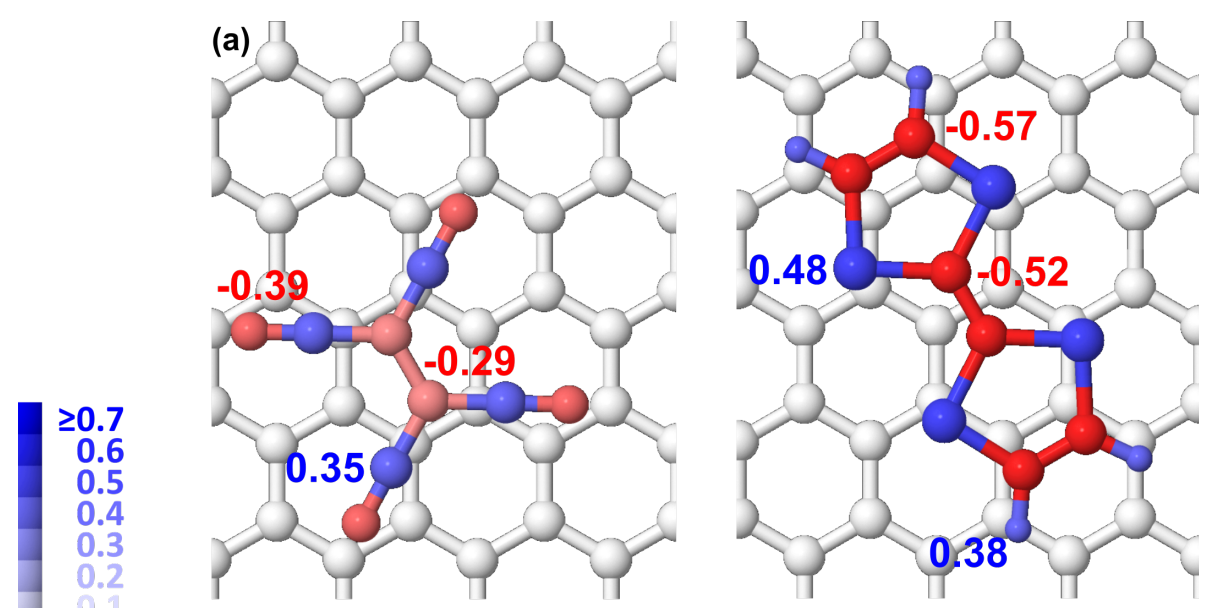

(0.0)
-0.1
-0.2
-0.3
-0.4
-0.5
-0.6
$\leq-0.7$
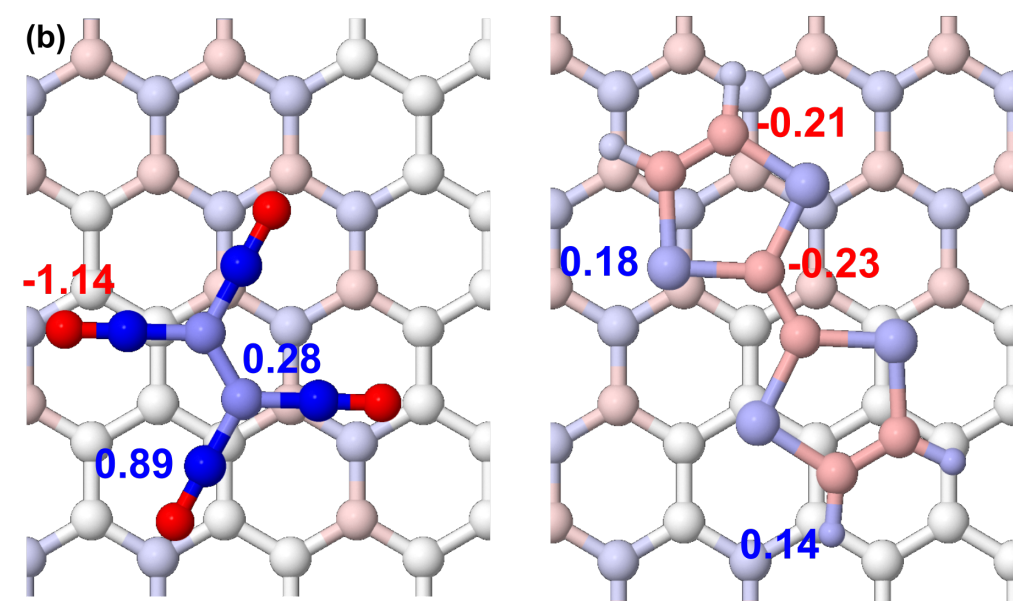

FIG. 1: Comparison of the AIM-Bader (bottom) and pMPA (top) partial atomic charges on EDAs (left: TCNE, right TTF). Atom colors are set up according to the charged-based color coded scheme plotted on the left.

As shown previously [12], if several adsorption sites presenting high symmetry can be tested, indeed they are almost degenerate, with energy differences smaller than $0.2 \mathrm{kcal} / \mathrm{mol}$. In this respect we propose here to discuss only the most stable state of adsorption for TTF and TCNE, starting for Bridge and Hollow geometries respectively, since the effect of the orientation of the molecule with respect to the graphene layer is really weak with CT differing by less than $0.02 \mathrm{e}$ at maximum between different adsorption sites. The selected optimized stacking geometries are depicted in Fig.1. We recall the importance of including van der Waals forces in the xc-functional term to yield correct adsorption height: with opt-B86b-DF TCNE lies $3.2 \AA$ above the graphene, while with PBE it is $3.38 \AA$. the same 
change is observed for the TTF molecule. The corresponding adsorption energies are in line with previous studies with values around $-30 \mathrm{kcal} / \mathrm{mol}$ when dispersion forces are included in the electronic correlation term. On top of these geometries we have performed AIMBader analysis, with various xc-functionals and also compared for two density decomposition schemes, namely AIM-Bader and pMPA.

\section{1. xc-functional}

In figure 2, TCNE and TTF CTs estimated by AIM-Bader scheme are shown for four selected xc-functionals on the same set of geometries. Surprisingly, for the acceptor molecule, the effect of using hybrid-type functional is weak, of the order of the integration's precision. Only RSHXLDA functional provides a slightly smaller CT probably due to its long-range exchange corrected HF term, compared to GGA estimates. So for this particular molecule, the estimates of CT by Bader analysis is more influenced by the distance between the molecule and graphene[12], and not primarily by the difference in the electronic structure of the molecule due to the use of hybrid functional, which usually enhances HOMO-LUMO gap. Indeed these values are 2.7, 3.7, 4.4 and $8.0 \mathrm{eV}$ for PBE, HSE, PBE0 and RSHXLDA respectively. In other words, space CT seems to be mainly governs by density overlaps between the two subsystems and globally the use of HF exchange term does not affect this overlap for the TCNE case. For the donor case, the situation is different, the PBE values is more than three times larger than with hybrid functionals, for the same molecule-graphene distance. When comparing the fundamental gaps for the freestanding TTF molecule which are 2.1, 3.1, 3.9 and $7.0 \mathrm{eV}$ for PBE, HSE, PBE0 and RSHXLDA respectively, one cannot find the origin of the discrepancy, the differences between GGA and hybrid values being very similar to the TCNE case. Here we propose that GGA tendency to over-delocalize electronic density already presented as a manifestation of self-interaction error (SIE), [51] is thus responsible for the $\mathrm{CT}$ differences. Indeed if one looks at figure 3a), one immediately sees that the use of PBE0 hybrid functional relocalizes density mainly around C-S bonds and S atoms for a freestanding TTF molecule. Upon adsorption this relocalization is more pronounced especially on $\mathrm{S}$ atoms. Assuming that the CT is mainly governed by density overlaps, since PBE suffers more from SIE for this particular test-molecule, PBE provides over-estimated $\mathrm{CT}$ values. 


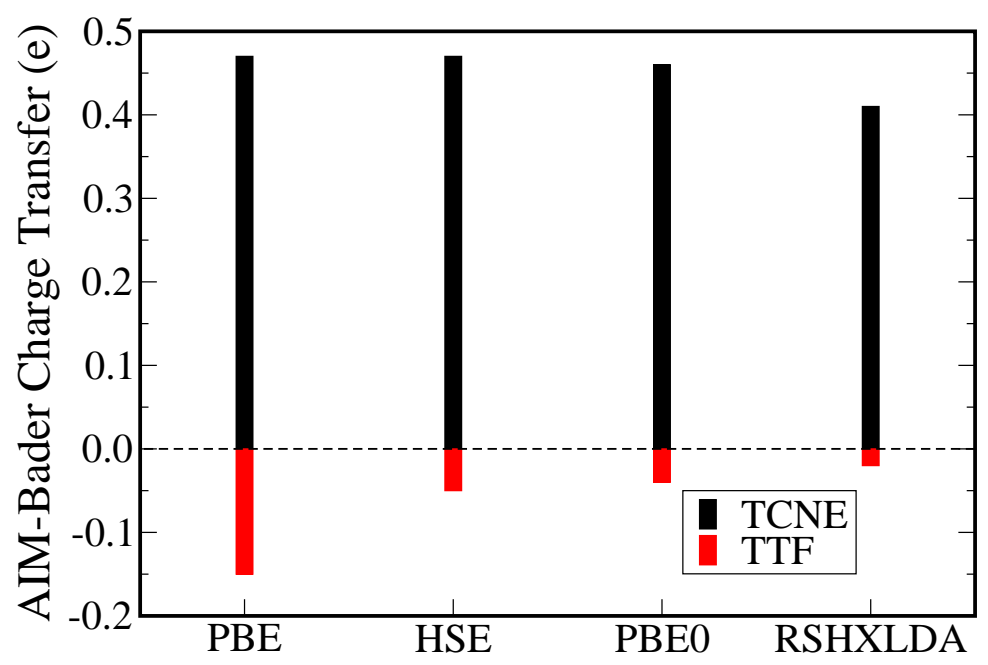

FIG. 2: AIM-Bader charge transfer with respect to xc-functional choice, for TCNE (black) and TTF (red) molecules.
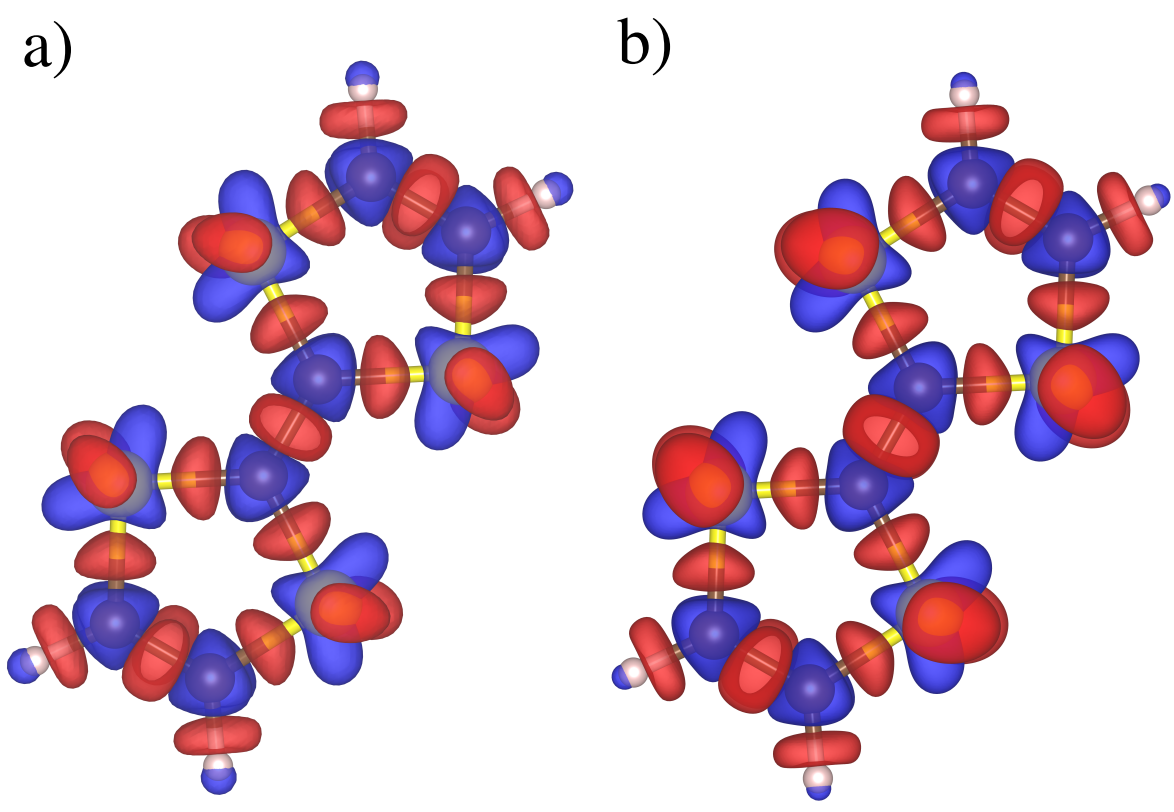

FIG. 3: Isovalues of electronic density differences $\left(\rho_{\mathrm{PBE} 0}-\rho_{\mathrm{PBE}}\right)$ of a $)$ a freestanding TTF molecule and b) an adsorbed TTF molecule on graphene. Positive isovalues $\left(+0.001 e / \AA^{3}\right)$ are in red, negative values are in blue $\left(-0.001 e / \AA^{3}\right)$. 


\begin{tabular}{c|cc|cc}
\hline & \multicolumn{2}{|c|}{ PBE } & \multicolumn{2}{c}{ PBE0 } \\
& TCNE TTF & TCNE TTF \\
\hline \hline pMPA & 0.68 & -0.21 & 0.74 & -0.12 \\
AIM & 0.47 & -0.15 & 0.46 & -0.04 \\
Magnetic moment & 0.44 & 0.00 & 0.44 & 0.00 \\
\hline
\end{tabular}

TABLE III: Charge transfer (in units of $e$ ) between EDA and graphene, and the final magnetic moment $\left(\right.$ in $\left.\mu_{B}\right)$.

In Table III, CT evaluations with pMPA and AIM for both molecules and two different xc-functionals are given. We also provides the final magnetic moment of the calculation cell, since it is a relevant physical property [43] and a direct manifestation of the CT. Indeed since a pristine graphene is diamagnetic, any charge changes (being positive or negative) on graphene do not affect the total magnetic moment, contrarily to the adsorbate. This indirect estimate of the $\mathrm{CT}$ is accurate if the adsorbed molecules do not interact strongly with the $\pi$ and $\pi^{*}$ orbitals of the graphene. Since TCNE and TTF molecules are physisorbed on graphene, one can be confident in this values. By comparing electron density partitioning schemes, the main comment is that pMPA approach always provides larger CT than AIM, and suffers from xc-functional's choice too. Regarding TTF, larger absolute values are yielded with PBE than with PBE0. Interestingly AIM CT values for the TCNE molecule are very close to the magnetic moment value for both functionals, when one can observe larger differences for the TTF molecule. From this table, one can indeed conclude that PBE overestimates the CT for TTF-Graphene system, since a zero magnetic moment is obtained for this system. Slightly more reliable results are obtained with the use of a hybrid functional, the PBE0 CT being -0.04e, making the CT much smaller for this donor molecule than for the acceptor TCNE molecule. Experimentally, CTs are observed in G-band shifts in Raman spectra for some graphene flakes in interaction with TTF solution [11], the shifts being smaller for TTF than for TCNE at the same concentration. Thus n-doping of graphene is much less efficient than p-doping, as observed experimentally. Moreover, in order to explain the discrepancy between the AIM CT value and the zero magnetic moment for the TTF molecule, one may suspect that some weak hybridization starts to occur between the 
adsorbate and the graphenic substrate, making the entire system diamagnetic.

Let us now compare the pMPA and AIM atomic charges within PBE0 calculations (Fig. 1). As already found in freestanding TCNE, ethylenic carbons are found to be negative with pMPA and positive with AIM. Whereas the CT is found to be fairly the same with both methods, they do not agree in the charge redistribution from graphene to TCNE. According to AIM the $\mathrm{CT}$ occurs mainly toward nitrogen atoms $(-1.14$ e vs. $-1.05 e$ in the freestanding TCNE),

whereas according to pMPA it is both nitrogen and ethylenic carbon atoms that benefit from the $\mathrm{CT}(\mathrm{C} / \mathrm{N}$ : $-0.29 e /-0.39 e$ vs. $-0.17 e /-0.27 e)$. Regarding now TTF, both methods find that the CT toward the graphene is weak, and the comparison between the charges given in Figure 3 and those reported in Table II shows so that there is not significant charge redistribution upon the adsorption of TTF on graphene.

\section{CONCLUSION}

To sum up, as already mentioned in a previous work, the primary interaction between an EDA molecule and a pristine graphene is of physisorption type. It appears thus natural to describe those systems with the help of an exchange-correlation functional that treats explicitly van der Waals forces. This imposes a correct distance between the adsorbate and the carbon-based substrate and at the same time authorize CT through space since electronic densities overlap. To estimate this CT we have considered two different schemes: one based on the AIM-Bader theory on one side and Mulliken-like scheme based on an atomic Slater basis set projection. AIM Bader charge transfer appear reliable for both donor and acceptor molecules, pMPA scheme seems to overestimate those CTs. However the intra-molecular partitioning are clearly different, for TCNE molecule for instance even some changes of sign are observed between the two schemes for the charge of two central $\mathrm{C}$ atoms. This makes the discussion of the extra-charge localization over the constituents of the molecule difficult. For the calculations considering the TTF molecule, the estimated $\mathrm{CT}$ is much weaker. When a portion of exact-exchange in included, this CT is even more decreased, making GGA calculations not reliable for this system. Globally this weak CT values is in line with experimental data, graphene is indeed less efficiently n-doped than p-doped, at the same concentration of dopants. More systematic studies, especially with 
others donor molecules, are thus further required. Out of these results, we can only advice to use both partitioning schemes, firstly to double-check the charge transfer values and secondly since they appear complementary in terms of extra possible analysis, one based of the topological aspects of the electronic density, the other scheme being based on a more natural molecular-orbitals like analysis.

\section{Acknowledgments}

I. C. Gerber and R. Poteau thanks the CALMIP initiative for the generous allocation of computational times, through the project p0812, as well as the GENCI-CINES, GENCIIDRIS and GENCI-CCRT for the A004096649 grant.

[1] K S Novoselov, A K Geim, S V Morozov, D Jiang, Y Zhang, S V Dubonos, I V Grigorieva, and A A Firsov. Electric Field Effect in Atomically Thin Carbon Films. Science, 306:666, October 2004.

[2] A K Geim and K S Novoselov. The rise of graphene. Nat Mater, 6:183, February 2007.

[3] S Das Sarma, Shaffique Adam, E H Hwang, and Enrico Rossi. Electronic transport in twodimensional graphene. Rev Mod Phys, 83(2):407-470, May 2011.

[4] Yuanbo Zhang, Yan-Wen Tan, Horst L Stormer, and Philip Kim. Experimental observation of the quantum Hall effect and Berry's phase in graphene. Nature, 438(7065):201-204, November 2005.

[5] K S Novoselov, A K Geim, S V Morozov, D Jiang, M I Katsnelson, I V Grigorieva, S V Dubonos, and A A Firsov. Two-dimensional gas of massless Dirac fermions in graphene. Nature, 438(7065):197-200, November 2005.

[6] Hongtao Liu, Yunqi Liu, and Daoben Zhu. Chemical doping of graphene. J Mat Chem, 21(10):3335-3345, 2011.

[7] Vasilios Georgakilas, Michal Otyepka, Athanasios B Bourlinos, Vimlesh Chandra, Namdong Kim, K Christian Kemp, Pavel Hobza, Radek Zboril, and Kwang S Kim. Functionalization of Graphene: Covalent and Non-Covalent Approaches, Derivatives and Applications. Chem Rev, 112(11):6156-6214, September 2012. 
[8] Konstantin Dirian, M Ángeles Herranz, Georgios Katsukis, Jenny Malig, Laura RodríguezPérez, Carlos Romero-Nieto, Volker Strauss, Nazario Martín, and Dirk M Guldi. Low dimensional nanocarbons - chemistry and energy/electron transfer reactions. Chem Sci, 4(12):4335$20,2013$.

[9] Vasilios Georgakilas, Jitendra N Tiwari, K Christian Kemp, Jason A Perman, Athanasios B Bourlinos, Kwang S Kim, and Radek Zboril. Noncovalent Functionalization of Graphene and Graphene Oxide for Energy Materials, Biosensing, Catalytic, and Biomedical Applications. Chem Rev, 116(9):5464-5519, May 2016.

[10] Wei Chen, Shi Chen, Dong Chen Qi, Xing Yu Gao, and Andrew Thye Shen Wee. Surface Transfer p-Type Doping of Epitaxial Graphene. J Am Chem Soc, 129(34):10418-10422, August 2007 .

[11] Rakesh Voggu, Barun Das, Chandra Sekhar Rout, and C N R Rao. Effects of charge transfer interaction of graphene with electron donor and acceptor molecules examined using Raman spectroscopy and cognate techniques. J Phys: Condens Matter, 20(47):472204-6, November 2008.

[12] Tao Hu and Iann C Gerber. Theoretical Study of the Interaction of Electron Donor and Acceptor Molecules with Graphene. J Phys Chem C, 117(5):2411-2420, January 2013.

[13] Liping Chen, Linjun Wang, Zhigang Shuai, and David Beljonne. Energy Level Alignment and Charge Carrier Mobility in Noncovalently Functionalized Graphene. J Phys Chem Lett, 4(13):2158-2165, June 2013.

[14] R F W Bader. Atoms in Molecules: A Quantum Theory. Inter Ser Monogr Chem. Clarendon Press, 1994.

[15] Arun K Manna and Swapan K Pati. Tuning the Electronic Structure of Graphene by Molecular Charge Transfer: A Computational Study. Chem Asian J, 4(6):855-860, June 2009.

[16] Y H Lu, W Chen, Y P Feng, and P M He. Tuning the Electronic Structure of Graphene by an Organic Molecule. J Phys Chem B, 113(1):2-5, January 2009.

[17] Yong-Hui Zhang, Kai-Ge Zhou, Ke-Feng Xie, Jing Zeng, Hao-Li Zhang, and Yong Peng. Tuning the electronic structure and transport properties of graphene by noncovalent functionalization: effects of organic donor, acceptor and metal atoms. Nanotechnology, 21(6):065201-8, January 2010.

[18] J T Sun, Y H Lu, W Chen, Y P Feng, and A T S Wee. Linear tuning of charge carriers 
in graphene by organic molecules and charge-transfer complexes. Phys Rev B, 81(15):176-6, April 2010.

[19] Mei Chi and Ya-Pu Zhao. First principle study of the interaction and charge transfer between graphene and organic molecules. Comp Mater Sci, 56(C):79-84, April 2012.

[20] Pablo A Denis. Chemical Reactivity of Electron-Doped and Hole-Doped Graphene. J Phys Chem C, 117(8):3895-3902, February 2013.

[21] Lingmei Kong, Axel Enders, Talat S Rahman, and Peter A Dowben. Molecular adsorption on graphene. J Phys: Condens Matter, 26(44):443001-28, October 2014.

[22] Pablo A Denis and Federico Iribarne. Strong N-Doped Graphene: The Case of 4-(1,3Dimethyl-2,3-dihydro-1 H-benzoimidazol-2-yl)phenyl)dimethylamine ( N-DMBI). J Phys Chem C, 119(27):15103-15111, June 2015.

[23] Kazume Nishidate, Noriyuki Yoshimoto, Peerasak Chantngarm, Hiroaki Saito, and Masayuki Hasegawa. Tuning the work function of graphene with the adsorbed organic molecules: firstprinciples calculations. Mol Phys, 114(20):2993-2998, 2016.

[24] Sha Yang, Yingda Jiang, Shuang Li, and Wei Liu. Many-body dispersion effects on the binding of TCNQ and F4-TCNQ with graphene. Carbon, 111(C):513-518, January 2017.

[25] G. Kresse and J. Hafner. Ab initio molecular dynamics for liquid metals. Phys Rev B, 47:558561, Jan 1993.

[26] G. Kresse and J. Furthmüller. Efficiency of ab-initio total energy calculations for metals and semiconductors using a plane-wave basis set. Comput Mater Sci, 6:15, 1996.

[27] P E Blöchl. Projector augmented-wave method. Phys Rev B, 50:17953, 1994.

[28] G Kresse and D Joubert. From ultrasoft pseudopotentials to the projector augmented-wave method. Phys Rev B, 59(3):1758-1775, 1999.

[29] Jiří Klimeš, David R Bowler, and Angelos Michaelides. Chemical accuracy for the van der Waals density functional. J Phys: Condens Matter, 22(2):022201, 2010.

[30] Jiří Klimeš, David R Bowler, and Angelos Michaelides. Van der Waals density functionals applied to solids. Phys Rev B, 83(24):195131, May 2011.

[31] John P. Perdew, Kieron Burke, and Matthias Ernzerhof. Generalized gradient approximation made simple. Phys Rev Lett, 77:3865-3868, Oct 1996.

[32] John P Perdew, M Ernzerhof, and Kieron Burke. Rationale for Mixing Exact Exchange with Density Functional Approximations. J Chem Phys, 105:9982-9985, November 1996. 
[33] Carlo Adamo and Vincenzo Barone. Toward Reliable Density Functional Methods without Adjustable Parameters: The PBE0 Model. J Chem Phys, 110(13):6158-6170, April 1999.

[34] J Heyd and G E Scuseria. Assessment and Validation of a Screened Coulomb Hybrid Density Functional. J Chem Phys, 120:7274, 2004.

[35] J Heyd, J E Peralta, G E Scuseria, and R L Martin. Energy Band Gaps and Lattice Parameters Evaluated with the Heyd- Scuseria-Ernzerhof Screened Hybrid Functional. J Chem Phys, $123: 174101,2005$.

[36] J Paier, M Marsman, K Hummer, G Kresse, I C Gerber, and Ángyán J. G. Screened Hybrid Density Functionals Applied to Solids. J Chem Phys, 124(15):154709, 2006.

[37] I C Gerber and János G Ángyán. Hybrid functional with separated range. Chem Phys Lett, 415:100, 2005.

[38] Iann C Gerber, János G Ángyán, Martijn Marsman, and Georg Kresse. Range separated hybrid density functional with long-range Hartree-Fock exchange applied to solids. $J$ Chem Phys, 127(5):054101-10, August 2007.

[39] Graeme Henkelman, Andri Arnaldsson, and Hannes Jónsson. A fast and robust algorithm for Bader decomposition of charge density. Comput Mater Sci, 36(3):354-360, 2006.

[40] Edward Sanville, Steven D Kenny, R Smith, and G Henkelman. Improved grid-based algorithm for Bader charge allocation. J Comput Chem, 28:899-908, 2007.

[41] W Tang, E Sanville, and G Henkelman. A grid-based Bader analysis algorithm without lattice bias. J Phys: Condens Matter, 21(8):084204-8, January 2009.

[42] Kenneth B Wiberg and Paul R Rablen. Comparison of atomic charges derived via different procedures. J Comput Chem, 14(12):1504-1518, December 1993.

[43] O Leenaerts, B Partoens, and F M Peeters. Paramagnetic adsorbates on graphene: A charge transfer analysis. Appl Phys Lett, 92(24):243125-4, June 2008.

[44] Volker L. Deringer, Andrei L. Tchougréeff, and Richard Dronskowski. Crystal orbital hamilton population (COHP) analysis as projected from plane-wave basis sets. J Phys Chem A, 115(21):5461-5466, 2011.

[45] Stefan Maintz, Volker L. Deringer, Andrei L. Tchougréeff, and Richard Dronskowski. Analytic projection from plane-wave and PAW wavefunctions and application to chemical-bonding analysis in solids. J Comput Chem, 34(29):2557-2567, 2013.

[46] Stefan Maintz, Volker L. Deringer, Andrei L. Tchougréeff, and Richard Dronskowski. LOB- 
STER: A tool to extract chemical bonding from plane-wave based DFT. J Comput Chem, 37(11):1030-1035, feb 2016.

[47] A. E. Reed, R. B. Weinstock, and F. Weinhold. Natural population analysis. J Chem Phys, 83:735-746, 1985 .

[48] Aleksandr V. Marenich, Steven V. Jerome, Christopher J. Cramer, and Donald G. Truhlar. Charge model 5: An extension of hirshfeld population analysis for the accurate description of molecular interactions in gaseous and condensed phases. J Chem Theor Comput, 8(2):527-541, feb 2012.

[49] M. J. Frisch, G. W. Trucks, H. B. Schlegel, G. E. Scuseria, M. A. Robb, J. R. Cheeseman, G. Scalmani, V. Barone, B. Mennucci, G. A. Petersson, H. Nakatsuji, M. Caricato, X. Li, H. P. Hratchian, A. F. Izmaylov, J. Bloino, G. Zheng, J. L. Sonnenberg, M. Hada, M. Ehara, K. Toyota, R. Fukuda, J. Hasegawa, M. Ishida, T. Nakajima, Y. Honda, O. Kitao, H. Nakai, T. Vreven, J. A. Montgomery, Jr., J. E. Peralta, F. Ogliaro, M. Bearpark, J. J. Heyd, E. Brothers, K. N. Kudin, V. N. Staroverov, R. Kobayashi, J. Normand, K. Raghavachari, A. Rendell, J. C. Burant, S. S. Iyengar, J. Tomasi, M. Cossi, N. Rega, J. M. Millam, M. Klene, J. E. Knox, J. B. Cross, V. Bakken, C. Adamo, J. Jaramillo, R. Gomperts, R. E. Stratmann, O. Yazyev, A. J. Austin, R. Cammi, C. Pomelli, J. W. Ochterski, R. L. Martin, K. Morokuma, V. G. Zakrzewski, G. A. Voth, P. Salvador, J. J. Dannenberg, S. Dapprich, A. D. Daniels, Ö. Farkas., J. B. Foresman, J. V. Ortiz, J. Cioslowski, and D. J. Fox. Gaussian 09 Revision D.01, 2009. Gaussian Inc. Wallingford CT.

[50] Tian Lu and Feiwu Chen. Multiwfn: A multifunctional wavefunction analyzer. J Comput Chem, 33(5):580-592, dec 2011.

[51] Xiao Zheng, Min Liu, Erin R Johnson, Julia Contreras-García, and Weitao Yang. Delocalization error of density-functional approximations: A distinct manifestation in hydrogen molecular chains. J Chem Phys, 137(21):214106-8, December 2012. 